\title{
Millimole per Liter per Milligram per Meter Squared
}

National Cancer Institute

\section{Source}

National Cancer Institute. Millimole per Liter per Milligram per Meter Squared. NCI

Thesaurus. Code C119416.

A unit of concentration equal to millimoles per liter, divided by milligrams per meter squared. 